\title{
AVALIAÇÃO DA DINÂMICA DO CULTIVO DA CANA-DE-AÇÚCAR NO ESTADO DE SÃ̃ PAULO POR MEIO DE PERFIS TEMPORAIS DE DADOS MODIS
}

Doi:http://dx.doi.org/10.1590/1809-4430-Eng.Agric.v35n6p 1127-1136/2015

\section{JOÃO F. G. ANTUNES ${ }^{1}$, RUBENS A. C. LAMPARELLI ${ }^{2}$, LUIZ H. A. RODRIGUES ${ }^{3}$}

RESUMO: As imagens do sensor MODIS fornecem dados que cobrem áreas de grande extensão com alta periodicidade, características fundamentais que possibilitam o monitoramento de culturas agrícolas estratégicas para o Brasil, como as da cana-de-açúcar. Técnicas matemáticas vêm sendo empregadas no estudo de longas séries temporais de índices de vegetação, baseado nas mudanças que acontecem na superfície terrestre, o que facilita o entendimento da dinâmica temporal. O objetivo deste trabalho foi realizar a avaliação da dinâmica do cultivo da cana-de-açúcar no Estado de São Paulo por meio de perfis temporais de dados MODIS, ao longo das safras de 2004/2005 a 2011/2012. A Transformada de Wavelet Daubechies 8 aplicada à série temporal do EVI2 do MODIS mostrou ser uma técnica robusta, pois conseguiu eliminar os ruídos, propiciando, assim, melhor captura das tendências dos ciclos de desenvolvimento da cana-de-açúcar em toda a série temporal. Os perfis temporais suavizados do EVI2 puderam ser utilizados no monitoramento do cultivo da cana-de-açúcar para identificar as épocas de mudanças do uso do solo e da cobertura da terra, acompanhando as variações sazonais dos ciclos fenológicos desde o plantio ou rebrota das soqueiras até à colheita.

PALAVRAS-CHAVE: sensoriamento remoto, processamento de imagens, índice de vegetação, séries temporais.

\section{ASSESSING OF THE SUGARCANE CULTIVATION DYNAMICS IN SÃO PAULO STATE BY MODIS DATA TEMPORAL PROFILES}

\begin{abstract}
MODIS sensor images provide data covering broad areas with high periodicity. Such features are fundamental for monitoring of strategic agricultural crops in Brazil, just as sugarcane. Mathematics techniques have been applied to time-series of remote sensing imagery to characterize vegetation phenology and to better understand its dynamics. This study aimed at assessing the dynamics of sugarcane cultivation throughout São Paulo State by means of MODIS data temporal profiles from 2004/2005 to 2011/2012 crop years. Daubechies 8 Wavelet Transformation applied to time-series MODIS EVI2 consists of a robust technique to remove noise, thereby providing a better capture of sugarcane cycle trends within the entire time-series. Smoothed EVI2 temporal profiles could be used for sugarcane cultivation monitoring and land cover change detection, accompanying seasonal variations in phenology from planting or ratoon regrowth until harvest.
\end{abstract}

KEYWORDS: remote sensing, image processing, vegetation index, time-series.

\section{INTRODUÇÃO}

O setor agrícola brasileiro foi marcado por um novo ciclo no plantio da cana-de-açúcar para obtenção do álcool combustível. Além de ser o maior produtor de cana-de-açúcar do mundo, o Brasil é, também, o maior exportador de açúcar e o segundo maior produtor de etanol. Metade da produção de açúcar é exportada, tendo a participação aproximada de $35 \%$ na balança comercial. Além disso, a agroindústria sucroalcooleira representa $6 \%$ dos empregos brasileiros. A região

\footnotetext{
${ }^{1}$ Eng $^{\circ}$ A grícola, Doutor, Pesquisador da Embrapa Informática Agropecuária, Fone: (19) 3211-5847, joao.antunes@embrapa.br

${ }^{2}$ Eng $^{\circ}$ de Transportes, Doutor, Pesquisador do Núcleo Interdisciplinar de Planejamento Energético - NIPE e Professor da Faculd ade de En genharia A grícola - Feagri, UNICAMP/ Campinas - SP, lamparel@unicamp.br

${ }^{3}$ Eng $^{\circ}$ A grícola, Prof. Doutor, Livre Docente da Faculdade de Engenharia A grícola - Feagri, UNICAMP/ Campinas - SP, lique@feagri.unicamp.br 
Centro-Sul do país é responsável por $90 \%$ do total da produção de cana-de-açúcar nacional, sendo o Estado de São Paulo o maior produtor, respondendo por mais de $60 \%$ de toda a produção de açúcar e de etanol (UNICA, 2014).

A cana-de-açúcar é uma gramínea da família Poáceae, e como pode ter vários ciclos de desenvolvimento, é caracterizada como uma cultura semiperene, permitindo uma média de cinco cortes para, posteriormente, ser feita a reforma do canavial. Possui um rápido crescimento, reprodução abundante e o aproveitamento econômico de grande parte da planta. O ciclo fenológico da cana-de- -açúcar é composto pelas fases de brotação, perfilhamento, crescimento e maturação (SCARPARI \& BEAUCLAIR, 2010).

Do plantio até à primeira colheita, a cana-planta pode ter um ciclo de desenvolvimento com duração de 18 meses (cana de ano e meio) ou 12 meses (cana de ano), dependendo da época e da região de plantio. Após a primeira colheita, a cana-soca passa a ter um ciclo de 12 meses, e a cada corte ocorre a rebrota da soqueira e o início de um novo ciclo. No Estado de São Paulo, predominam os ciclos de produção da cana de ano e meio, plantada de janeiro a maio, e da cana de ano, plantada de setembro a dezembro, com a colheita estendendo-se de abril a dezembro (ANJOS \& FIGUEIREDO, 2010).

Os satélites de monitoramento ambiental vêm operando de forma sistemática e ininterrupta nos últimos anos, provendo uma visão sinóptica e frequente de grandes áreas, a custos baixos. As imagens geradas pelos sensores orbitais dentro desses satélites têm características que possibilitam o estudo e a análise do ciclo de desenvolvimento de culturas agrícolas (VICENTE et al., 2012).

O sensor MODIS (Moderate Resolution Imaging Spectroradiometer), a bordo das plataformas orbitais do programa internacional EOS (Earth Observing System), liderado pela NASA (National Aeronautics and Space Administration), tem gerado dados processados para estudos globais da vegetação. O satélite TERRA foi lançado em dezembro de 1999 e tem passagem pelo Equador às $10 \mathrm{~h} 30$ (horário local), em órbita descendente (SOARES et al., 2007).

Os dados MODIS de moderada resolução espacial, elevada repetitividade temporal, boa qualidade radiométrica, alta precisão geométrica, com correção atmosférica e de distribuição gratuita, possuem características que proporcionam um grande potencial de aplicação no monitoramento da cultura da cana-de-açúcar (ADAMI et al., 2012).

Os índices de vegetação extraídos dos dados MODIS realçam a resposta espectral das plantas e amenizam a influência do solo, com o propósito de distinguir a fenologia da vegetação e as alterações do uso e da cobertura da terra na análise ao longo de uma série temporal de imagens. Das imagens sequenciais dos índices de vegetação, podem ser gerados os perfis temporais, que descrevem o comportamento espectral característico de alvos localizados na superfície terrestre ao longo do tempo (BROWN et al., 2013).

Nesse contexto, o objetivo do trabalho foi realizar a avaliação da dinâmica do cultivo da cana-de-açúcar no Estado de São Paulo por meio de perfis temporais de dados MODIS, ao longo das safras de 2004/2005 a 2011/2012.

\section{MATERIAL E MÉTODOS}

Os perfis temporais foram gerados a partir das séries temporais de índ ices de vegetação, em 10 áreas de cultivo da cana-de-açúcar no Estado de São Paulo, referentes aos dados de campo fornecidos pela Raízen, que é uma das maiores empresas do setor de energia do Brasil, com o plantio realizado em terras arrendadas, e 24 unidades produtoras de cana-de-açúcar distribuídas pelo país. Porém, a possível presença de ruídos nas composições MODIS pode afetar o reconhecimento do padrão espectral característico da cana-de-açúcar ao longo das safras.

A Transformada de Wavelet foi utilizada com a finalidade de suavizar os perfis temporais, fazendo o ajuste de uma curva para remover os ruídos de alta frequência e destacar as alterações de baixa frequência relacionadas à fenologia da vegetação. Segundo MARTíNEZ \& GILABERT (2009), essa transformação matemática é aplicada para decompor e recompor dados em diferentes 
escalas temporais, para obtenção de informações ocultas no domínio da frequência, em que cada escala é representada por uma frequência específica.

Com base em DAUBECHIES (2006), uma série temporal pode ser representada pela Transformada de Wavelet, sendo $\phi(t)$ uma função de oscilação com energia finita e média nula, conforme a [eq. (1)]:

$$
\int_{-\infty}^{+\infty} \phi(t) d t=0
$$

A Transformada de Wavelet W(a,b) é definida pela [eq. (2)]:

$$
\mathrm{W}(\mathrm{a}, \mathrm{b})_{\mathrm{i}}=\frac{1}{\sqrt{|\mathrm{a}|}} \int \phi^{*}\left(\frac{\mathrm{t}-\mathrm{b}}{\mathrm{a}}\right) \mathrm{s}(\mathrm{t}) \mathrm{dt}
$$

em que,

$\mathrm{s}(\mathrm{t})$ é o sinal de entrada analisado, e

$\phi^{*}$ é a wavelet mãe ou base.

Nesta equação, a largura da wavelet é determinada pelo parâmetro de escala "a", enquanto seu centro é determinado pelo parâmetro " $b$ ", com $a, b \in R$ e $a \neq 0$. Para trabalhar com sinais discretos, usualmente se utilizam valores especiais para a e $b$, sendo $a=2^{j} e b=k 2^{j}, \operatorname{com} j, k \in Z$. Mudando o valor do parâmetro "a", tem-se o efeito de dilatação $(a>1)$ ou de contração $(a<1)$, enquanto mudanças no parâmetro "b" têm o efeito de translação para analisar a função $\phi(t)$ em torno desse ponto. A variável " $t$ " representa o intervalo de tempo na série temporal, na qual é realizada a integração. A Transformada de Wavelet tem a vantagem de manter as informações relacionadas à largura (escala) e à localização (tempo) das características presentes em s(t). Conforme GENDRIN et al. (2006), a reconstrução do sinal pode ser realizada pela [eq. (3)]:

$$
\mathrm{W}=\sum_{\mathrm{i}=1}^{\mathrm{x}} \mathrm{W}(\mathrm{a}, \mathrm{b})_{\mathrm{i}}
$$

A série temporal de wavelet $\mathrm{W}$ é um somatório das wavelets de larguras decrescentes de i a $\mathrm{x}$, em que x é número de transformações necessárias para alcançar o número definido de coeficientes retidos dos dados de entrada. A filtragem inicia-se com a aplicação da função de suavização na série temporal para remover os ruídos de alta frequência. A série temporal suavizada é então reconstruída com a Transformada de Wavelet discreta. As funções wavelets são obtidas por uma dilatação e uma translação, formando uma base que não precisa ser necessariamente ortogonal. Uma das vantagens de se trabalhar com funções de bases ortogonais é que permitem a reconstrução perfeita do sinal original a partir dos coeficientes das wavelets (MORETTIN, 1999).

A aplicação da Transformada de Wavelet, numa série temporal, requer a definição dos parâmetros ordem e potência da wavelet mãe que definem o comportamento da curva. Ordem é uma medida de suavidade, em que valores mais elevados produzem mais suavização. Potência corresponde ao número de coeficientes retidos da série temporal original. Maior potência gera uma wavelet mais estreita que inclui detalhes de escala mais fina, mas também pode reter mais ruído. Menor potência remove mais ruído pela forma mais larga da wavelet, captura tendências, mas perde detalhes fenológicos (GALFORD et al., 2008). 
A Figura 1 ilustra a função wavelet mãe e a escala da família Daubechies 8 (Db8). Por ser ortogonal, essa função assegura que o sinal decomposto é reconstruído sem a presença de res íduos devido à assimetria da wavelet mãe.

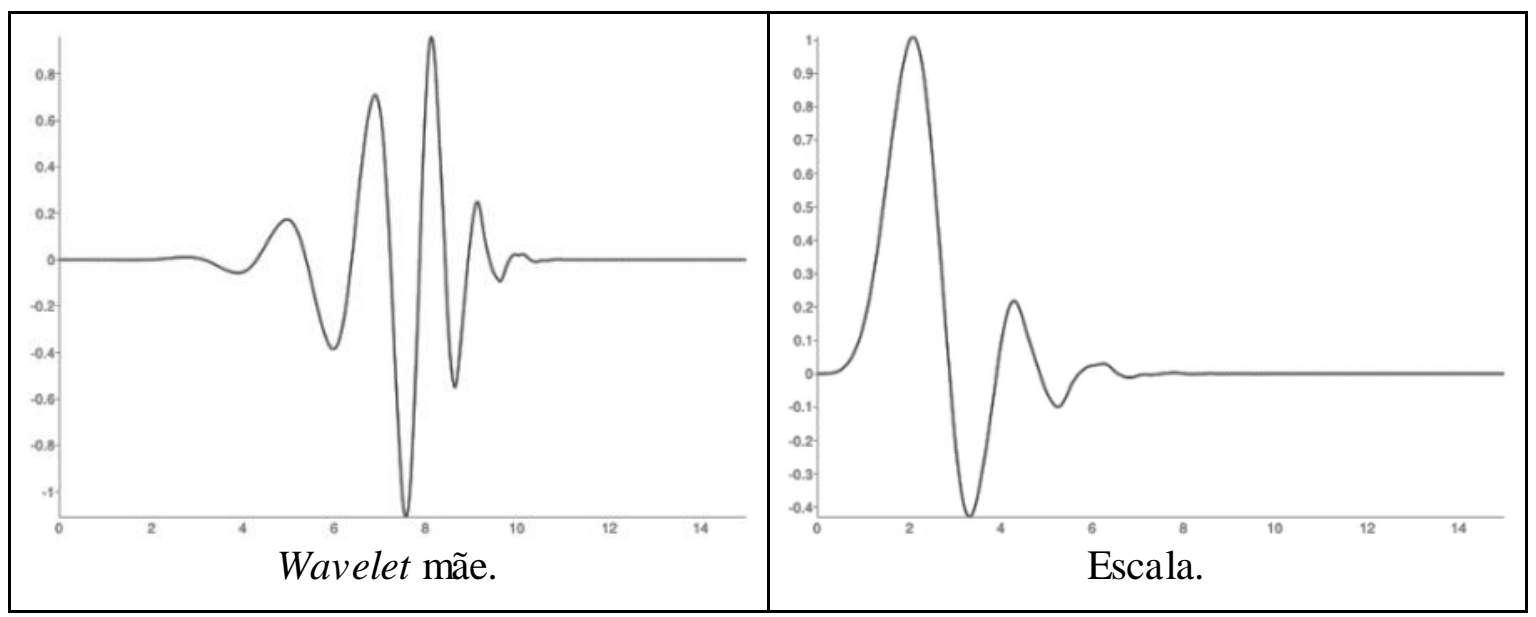

FIGURA 1. Wavelet da família Daubechies 8. Daubechies 8 wavelet family. Fonte: WASILEWSKI (2014).

Os perfis temporais suavizados dos dados MODIS foram gerados por meio do sistema Series View do Instituto Nacional de Pesquisas Espaciais (INPE), que é um ambiente para a visualização de séries temporais para dar suporte a estudos e a análises de mudança de uso e de cobertura da terra (INPE, 2014), mostrado na Figura 2.

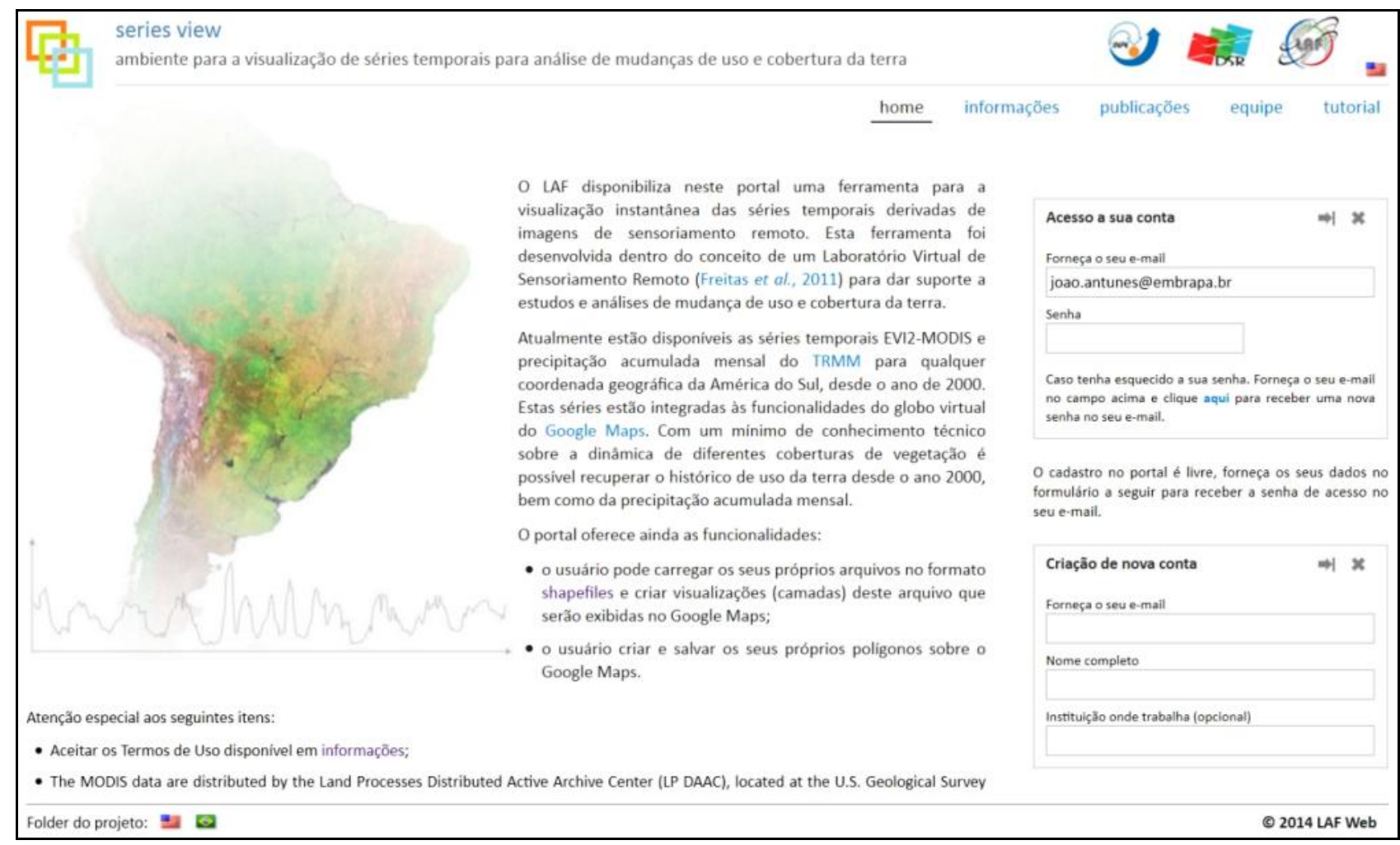

FIGURA 2. Ambiente para visualização de séries temporais MODIS. Environment for MODIS time-series visualization.

Fonte: INPE (2014).

Essa ferramenta gera, instantaneamente perfis, temporais a partir do índice de vegetação EVI2, calculado com dados das bandas espectrais das composições de 16 dias do MODIS, apresentando as curvas originais e as curvas suavizadas com a Transformada de Wavelet Daubechies (FREITAS et al., 2011). O EVI2, desenvolvido por JIANG et al. (2008), não utiliza a 
banda do azul por ser altamente influenciada pelos aerossóis da atmosfera. Deste modo, reduz os ruídos nas imagens, mostrando ter um comportamento muito similar ao EVI (Enhanced Vegetation Index), com o propósito de otimizar a resposta espectral da vegetação e melhorar a sensibilidade em regiões de maior biomassa. O EVI2 pode representar melhor a dinâmica da vegetação ao longo de uma série temporal de imagens e é calculado pela [eq. (4)], variando de - 1,0 a +1,0:

$$
\mathrm{EVI} 2=2,5 \times\left[\frac{\left(\rho_{\mathrm{IVP}}-\rho_{\mathrm{VER}}\right)}{\left(\rho_{\mathrm{IVP}}+2,4 \times \rho_{\mathrm{VER}}+1\right)}\right]
$$

em que,

$\rho_{\text {VER }}=$ fator de refletância na banda do vermelho

$\rho_{\mathrm{IVP}}=$ fator de refletância na banda do infravermelho próximo.

A curva gerada pela Transformada Wavelet Daubechies 8 propicia capturar as tendências dos ciclos vegetativos em toda a série temporal. No entanto, cabe ressaltar que a suavização acentuada pode provocar diminuição dos valores mais altos do EVI2 e, assim, perder alguns detalhes da fenologia da vegetação.

As áreas de cultivo da cana-de-açúcar de referência, representadas na Figura 3, localizam-se nos seguintes municípios no Estado de São Paulo:

1) Igarapava $\left(20^{\circ} 00^{\prime} \mathrm{S}, 47^{\circ} 51^{\prime} \mathrm{O}\right)$ : Mesorre gião de Ribeirão Preto.

2) Aramina ( $\left.20^{\circ} 11^{\prime} \mathrm{S}, 47^{\circ} 49^{\prime} \mathrm{O}\right)$ : Mesorregião de Ribeirão Preto.

3) Guararapes $\left(21^{\circ} 09^{\prime} \mathrm{S}, 50^{\circ} 44^{\prime} \mathrm{O}\right)$ : Mesorre gião de Araçatuba.

4) Valparaíso $\left(21^{\circ} 22^{\prime} \mathrm{S}, 51^{\circ} 04^{\prime} \mathrm{O}\right)$ : Mesorregião de Araçatuba.

5) Araraquara $\left(21^{\circ} 48^{\prime} \mathrm{S}, 48^{\circ} 20^{\prime} \mathrm{O}\right)$ : Mesorre gião de Araraquara.

6) Boa Esperança do Sul (21 $\left.{ }^{\circ} 59^{\prime} \mathrm{S}, 48^{\circ} 27^{\prime} \mathrm{O}\right)$ : Mesorregião de Araraquara.

7) Itapuí ( $\left.22^{\circ} 16^{\prime} \mathrm{S}, 48^{\circ} 44^{\prime} \mathrm{O}\right)$ : Mesorregião de Bauru.

8) São Manuel $\left(22^{\circ} 33^{\prime} \mathrm{S}, 48^{\circ} 28^{\prime} \mathrm{O}\right)$ : Mesorregião de Bauru.

9) Santa Bárbara D’Oeste (22 $\left.50^{\prime} \mathrm{S}, 4^{\circ} 26^{\prime} \mathrm{O}\right)$ : Mesorregião de Campinas.

10) Tietê $\left(23^{\circ} 06^{\prime} S, 47^{\circ} 38^{\prime} \mathrm{O}\right)$ : Mesorregião de Piracicaba.

Os municípios de Igarapava, Aramina e Tietê estão localizados em regiões tradicionais de intensificação do cultivo da cana-de-açúcar. Já os municípios de Guararapes, Valparaíso, Araraquara, Boa Esperança do Sul, Itapuí e São Manuel estão localizados em regiões que apresentam forte expansão no cultivo da cana-de-açúcar. O município de Santa Bárbara D’Oeste localiza-se numa região de cultivo expressivo da cana-de-açúcar.

Todas as áreas de cultivo possuem condições favoráveis para o monitoramento da cana-deaçúcar, devido à predominância de relevo suavemente ondulado e clima tropical quente e úmido, permitindo o crescimento da planta durante a primavera e o verão, e oferecendo condições adequadas para a maturação e a colheita durante o outono e o inverno.

\section{RESULTADOS E DISCUSSÃO}

A Figura 3 apresenta a localização geográfica no sistema Series View das 10 áreas de cultivo da cana-de-açúcar de referência no Estado de São Paulo, de aproximadamente 400 hectares cada uma. 


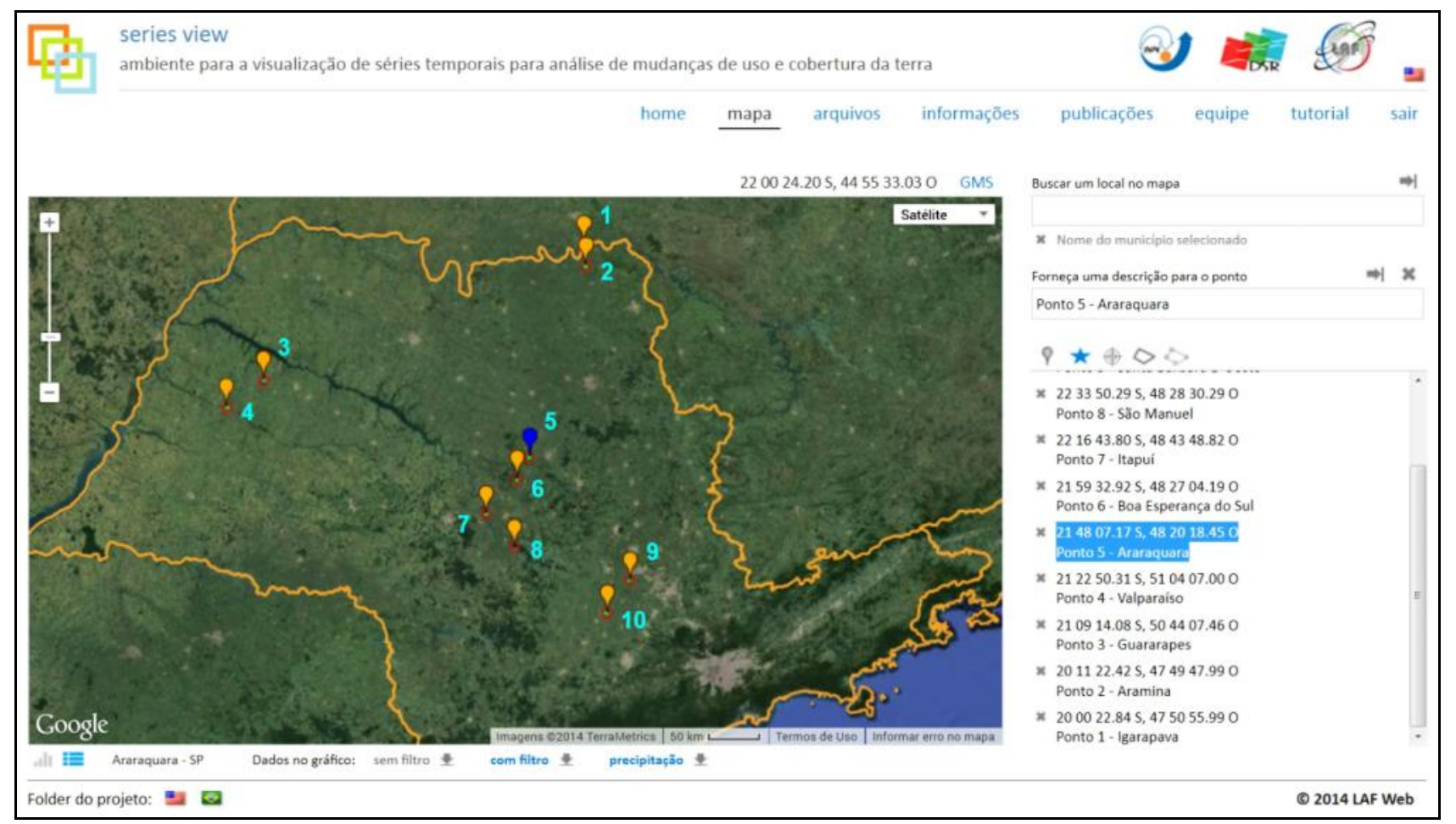

FIGURA 3. Áreas de cultivo da cana-de-açúcar de referência. Reference areas of sugarcane Fonte: INPE (2014).

\section{cultivation.}

O pixel selecionado para compor a série temporal corresponde ao centroide geométrico de cada área de cultivo, referente às coordenadas geográficas que estão entre parênteses, representando, as sim, o perfil espectro-temporal característico da respectiva área. Com base nisso, os perfis temporais do EVI2 do MODIS foram gerados no sistema Series View do INPE para cada uma das 10 áreas de cultivo da cana-de-açúcar de referência, conforme apresentado na Figura 4.

A curva em azul, na Figura 4, representa os dados originais do EVI2 com a presença de ruídos observados em um pixel das imagens MODIS ao longo do tempo. A curva em vermelho é resultado da suavização, utilizando a Transformada de Wavelet Daubechies 8.

Com base na análise dos gráficos da Figura 4, pode-se observar que a fenologia das áreas de cultivo da cana-de-açúcar apresenta grandes variações do EVI2, relacionada à alta dinâmica espectral da cana-de-açúcar durante o ano, em que a cultura é caracterizada por picos na série temporal, com predomínio do ciclo da cana de ano. Entre os meses de agosto e novembro, a cana-de-açúcar está na fase de perfilhamento, em que a planta se desenvolve de maneira abundante, mas os valores do EVI2 ainda são baixos. Quando se inicia a fase de crescimento, a partir de dezembro, os valores do EVI2 aumentam fortemente, devido ao alongamento dos colmos e ao acúmulo de matéria seca. A partir de abril, os valores do EVI2 decrescem na fase de maturação, que corresponde à senescência da planta, quando se inicia a colheita. 


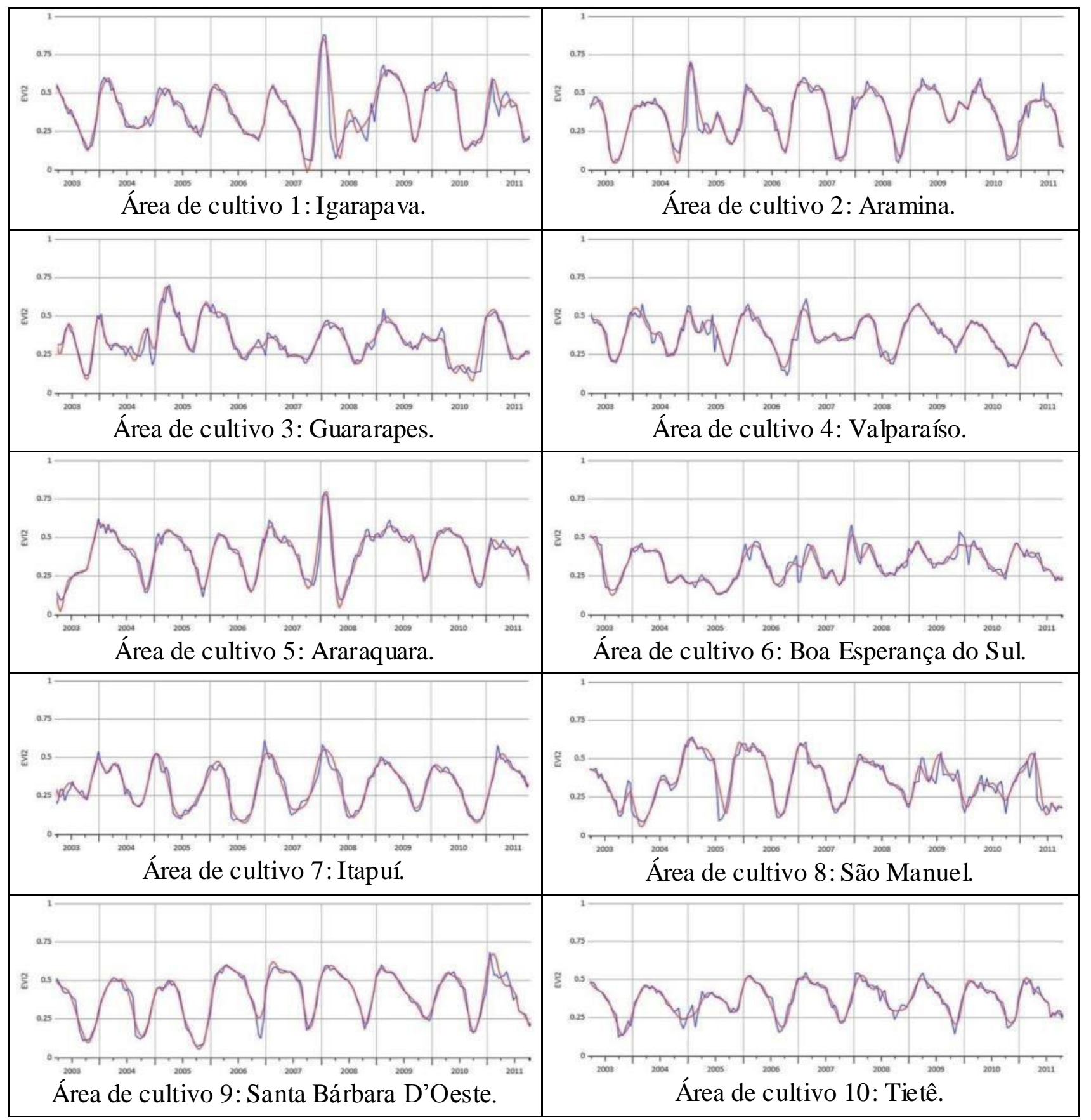

FIGURA 4. Perfis temporais do EVI2 nas áreas de cultivo da cana-de-açúcar de referência. Temporal EVI2 profiles in the reference areas of sugarcane cultivation.

A área 1, em Igarapava, e a área 5, em Araraquara, apresentam padrões semelhantes, podendo-se observar quatro ciclos de desenvolvimento de 2003 a 2007, iniciado com o plantio da cana de ano e meio e a rebrota da cana de ano a cada corte. A reforma do canavial ocorreu no final de 2007, identificada por um pico acentuado do EVI2, provavelmente devido ao cultivo de uma forrageira, seguida por mais três ciclos de desenvolvimento, da cana de ano e meio em 2008 e a rebrota da cana de ano a cada corte, em 2010 e 2011.

$\mathrm{Na}$ área 2 em Aramina, pode-se observar um ciclo da cana de ano em 2004 com a reforma do canavial, no início de 2005, seguida por seis ciclos da cana de ano.

$\mathrm{Na}$ área 3 em Guararapes e na área 4 em Valparaíso, podem-se ver três ciclos de desenvolvimento da cana de ano, de 2004 a 2006, a reforma do canavial no início de 2007, seguida por um ciclo da cana de ano e meio em 2008 e a rebrota da cana de ano a cada corte, de 2009 a 2011. 
A área 6, em Boa Esperança do Sul, apresenta alta variabilidade do EVI2, causada por uma grande mistura espectral inerente da fisiologia da cana-de-açúcar, em que é possível observar um ciclo da cana de ano em 2004, com uma provável reforma do canavial em 2005, seguida por seis ciclos da cana de ano, que não estão bem caracterizados pelo perfil temporal.

Na área 7 em Itapuí, na área 9 em Santa Bárbara D’Oeste e na área 10 em Tietê, podem-se perceber oito ciclos de desenvolvimento da cana de ano, de 2003 a 2011.

Na área 8 em São Manuel, pode-se notar a reforma do canavial no final de 2003, seguida por sete ciclos de desenvolvimento, da cana de ano e meio em 2004 e a rebrota da cana de ano a cada corte, entre 2006 e 2011.

Como dito anteriormente, uma das principais vantagens que as imagens do sensor MODIS apresentam é a alta repetitividade de extensas áreas da superfície terrestre, aliado à boa qualidade radiométrica e à excelente precisão geométrica. Essas são características fundamentais para o estudo de alvos com grande dinâmica espectral e temporal e, dessa forma, podem ser empregadas no monitoramento ao longo das fases do ciclo de desenvolvimento da cana-de-açúcar, desde o plantio ou rebrota até a colheita, em nível regional e local.

A abordagem espectro-temporal tem sido apontada como sendo a mais apropriada para monitorar e mapear culturas agrícolas por meio da análise do comportamento dos pixels das imagens, ao longo de uma série temporal, com ênfase nas variações espectrais. Dessa forma, é possível identificar a dinâmica da vegetação em distintas escalas temporais, acompanhando as variações dos ciclos fenológicos e caracterizando a transição entre diferentes usos e coberturas do solo ao longo dos anos (AGUIAR et al., 2009).

Entretanto, uma série temporal oriunda das imagens do sensor MODIS pode estar sujeita à interferência de ruídos relacionados principalmente à presença de nuvens, condições de iluminação e geometria de visada, mesmo em composições dos pixels com a melhor informação espectral dentro de um período de tempo (HIRD \& MCDERMID, 2009).

Neste sentido, o comportamento temporal de um índice de vegetação tende a ser equilibrado e de baixa frequência, mas as oscilações de alta frequência devem ser tratadas como ruídos, que podem ser reduzidos com a aplicação de técnicas matemáticas de análise de séries temporais, sem perder detalhes de fenologia da vegetação (RAMME et al., 2010).

Como qualquer fenômeno natural, o ciclo fenológico da cana-de-açúcar está relacionado com as condições climáticas, variando de acordo com as fases de desenvolvimento. Em função disso, em estudos futuros, sugere-se investigar a relação entre os índices de vegetação do sensor MODIS e a sazonalidade da precipitação, que pode indicar o potencial produtivo da cana-de-açúcar em termos de biomassa, permitindo comparar as condições atuais com as condições apresentadas em safras passadas.

\section{CONCLUSÕES}

Os resultados deste trabalho mostraram o potencial do monitoramento temporal na detecção de padrões de fenologia da cultura da cana-de-açúcar, cujo entendimento da dinâmica temporal é importante para se verificar suas variações interanuais.

A Transformada de Wavelet Daubechies 8 aplicada à série temporal do EVI2 do MODIS mostrou ser uma técnica robusta, pois conseguiu eliminar os ruídos, propiciando, assim, melhor captura das tendências dos ciclos de desenvolvimento da cana-de-açúcar em toda a série temporal.

Os perfis temporais suavizados do EVI2 puderam ser utilizados no monitoramento do cultivo da cana-de-açúcar para identificar as datas de mudanças do uso do solo e cobertura da terra, acompanhando as variações sazonais dos ciclos fenológicos desde o plantio ou rebrota das soqueiras até à colheita. 


\section{REFERÊNCIAS}

ADAMI, M.; MELLO, M. P.; AGUIAR, D. A.; RUDORFF, B. F. T.; SOUZA, A. F. A Web platform development to perform thematic accuracy asse ssment of sugarcane mapping in SouthCentral Brazil. Remote Sensing, Basel, v.4, n.10, p.3201-3214, 2012.

AGUIAR, D. A. de; RUDORFF, B. F. T.; ADAMI, M.; SHIMABUKURO, Y. E. Imagens de sensoriamento remoto no monitoramento da colheita da cana-de-açúcar. Engenharia Agrícola, Jaboticabal, v.29, n.3, p.440-451, 2009.

ANJOS, I. A. dos.; FIGUEIREDO, P. A. M. de. Aspectos fitotécnicos do plantio. In: DINARDOMIRANDA, L. L.; VASCONCELOS, A. C. M. de; LANDELL, M. G. de A. (Ed.). Cana-de-açúcar. Campinas: Instituto Agronômico, 2010. 882p.

BROWN, J. C.; KASTENS, J. H.; COUTINHO, A. C.; VICTORIA, D. de C.; BISHOP, C. R. Classifying multiyear agricultural land use data from Mato Grosso using time-series MODIS vegetation index data. Remote Sensing of Environment, New York, v.130, p.39-50, 2013.

DAUBECHIES, I. Ten lectures on wavelets. $9^{\text {th }}$. ed. Philadelphia: Rutgers University and AT\&T Bell Laboratories, 2006. 357p.,

FREITAS, R. M.; ARAI, E.; ADAMI, M.; SOUZA, A. F.; SATO, F. Y.; SHIMABUKURO, Y. E.; ROSA, R. R.; ANDERSON, L. O.; RUDORFF, B. F. T. Virtual laboratory of remote sensing time series: visualization of MODIS EVI2 data set over South America. Journal of Computational Inte rdisciplinary Sciences, Berlin, v.2, n.1, p.57-68, 2011.

GALFORD, G. L.; MUSTARD, J. F.; MELILLO, J.; GENDRIN, A.; CERRI, C. C., CERRI, C. E. P. Wavelet analysis of MODIS time series to detect expansion and intensification of row-crop agriculture in Brazil. Remote Sensing of Environment, New York, v.112, n.2, p.576-587, 2008.

GENDRIN, A.; LANGEVIN, Y.; BIBRING, J. P.; FORNI, O. A new method to investigate hyperspectral image cubes: an application of the wavelet transform. Journal of Geophysical Research, Richmond, v.111, n. E10, E10004, 21p., 2006.

HIRD, J. N.; MCDERMID, G. J. Noise reduction of NDVI time series: an empirical comparison of selected techniques. Remote Sensing of Environment, New York, v.113, n. 1, p.248-258, 2009.

INPE - INSTITUTO NACIONAL DE PESQUIS AS ESPACIAIS. Series view. Disponível em: <https://www.dsr.inpe.br/laf/series/>. Acesso em: 6 jul. 2014.

JIANG, Z.; HUETE, A. R.; DIDAN, K.; MIURA, T. Development of a two-band enhanced vegetation index without a blue band. Remote Sensing of Environment, New York, v.112, n.10, p.3833-3845, 2008.

MARTÍNEZ, B.; GILABERT, M. A. Vegetation dynamics from NDVI time series analysis using the wavelet transform. Remote Sensing of Environment, New York, v.113, n.9, p.1823-1842, 2009.

MORETTIN, P. A. Ondas e ondaletas: da análise de Fourier à análise de ondaletas. São Paulo: Editora da Universidade de São Paulo, 1999. 276p.

RAMME, F. L. P.; LAMPARELLI, R. A. C.; ROCHA, J. V. Perfis temporais NDVI MODIS, na cana-soca, de maturação tardia. Engenharia Agrícola, Jaboticabal, v.30, n.3, p.480-494, 2010.

SCARPARI, M. S.; BEAUCLAIR, E. G. F. de. Anatomia e botânica. In: DINARDO-MIRANDA, L. L.; VASCONCELOS, A. C. M. de; LANDELL, M. G. de A. (Ed.). Cana-de-açúcar. Campinas: Instituto Agronômico, 2010. 882p.

SOARES, J. V.; BATISTA, G. T; SHIMABUKURO. Y. E. Sensor MODIS: histórico e descrição. In: SHIMABUKURO, Y. E.; RUDORFF, B. F. T.; CEBALLOS, J. C. (Coord.). O Sensor MODIS e suas aplicações ambientais no Brasil. São José dos Campos: Editora Parêntese, SP, Brasil, 2007. $448 \mathrm{p}$. 
UNICA - UNIÃO DA AGROINDÚSTRIA CANAVIEIRA DE SÃO PAULO. UNICADATA. Disponível em: <http://www.unicadata.com.br/>. Acesso em: 30 jun. 2014.

VICENTE, L. E.; GOMES, D.; VICTORIA, D. de C.; GARÇOM, E. A. M.; BOLFE, E. L.; ANDRADE, R. G.; SILVA, G. B. S. Séries temporais de NDVI do sensor SPOT Vegetation e algoritmo SAM aplicados ao mapeamento de cana-de-açúcar. Pesquisa Agropecuária B rasileira, Brasília, v.47, n.9, set., p.1337-1345, 2012.

WASILEWSKI, F. PyWavelets: wavelet properties browser. Disponível em: <http://Wavelets.pybytes.com>. Acesso em: 6 jul. 2014. 